\title{
A GEOMETRIC VIEW OF WELFARE GAINS WITH NON-INSTANTANEOUS ADJUSTMENT
}

\author{
Peter SALAMON \\ Department of Mathematical Sciences, San Diego State University, San Diego, CA 92182, U.S.A. \\ John KOMLOS* \\ Carolina Population Center, University of North Carolina, Chapel Hill, NC 27514, U.S.A.
}

\author{
Bjarne ANDRESEN $\dagger$ and James D. NULTON $\ddagger$ \\ Department of Mathematical Sciences, San Diego State University, San Diego, CA 92182, U.S.A.
}

Communicated by K.H. Kim

Received 17 February 1986

\begin{abstract}
A geometric approach which defines distance between equilibrium states has proved useful in the physical sciences. The notion is applicable to any system which exhibits optimizing behavior and which does not adjust its states instantaneously to exogenous shocks. In this paper this geometry is used to analyze an economic system perturbed out of equilibrium by discrete or continuous shocks in order to establish in-principle limits to welfare gains generated by adjustments. The geometry is defined by the second derivative of the utility function which is used as the metric matrix. The minimum net gain in utility due to adjustment is proportional to the square of the distance traversed as measured in this geometry. An integrated welfare loss measuring the welfare losses due to the non-instantaneous response is defined. Finally, the geometry of the Cobb-Douglas utility function is explored.
\end{abstract}

Key words: Geometric economics; welfare gains; adjustment.

\section{Introduction}

Recent developments in thermodynamics (Salamon and Berry, 1983) as well as in the theory of communication (Flick et al., 1987) have demonstrated the feasibility of defining the notion of distance ${ }^{1}$ between two equilibrium states in a comparative statics framework. Rather than being restricted to its traditional role in the analysis

\footnotetext{
* Also affiliated with the Department of Economics, Duke University, Durham, NC 27706, U.S.A.

† Permanent address: Physics Laboratory, University of Copenhagen, Universitetsparken 5, DK-2100 ('openhagen $\emptyset$, Denmark.

$\ddagger$ Permanent address: Mathematics Department, San Diego City College, San Diego, CA 92101, U.S.A.

'Our use of 'distance' is not to be confused with the distance function defined as the dual of the cost function discussed in Deaton (1979).
} 
of stability, the quadratic form associated with the second derivative matrix of the objective function is used to define a metric structure on the set of equilibrium states. The length corresponding to a time evolution has important implications for the accompanying losses. In thermodynamics these losses are measured by the entropy produced or by the loss of available work. In communications theory these losses are measured by the extra number of bits which need to be transmitted through a communication channel. In general the metric structure can be used to measure losses associated with the inability of a system to adjust instantaneously to exogenous shocks.

Consider a system whose equilibrium states optimize some objective function. By the conditions of optimality, a slight displacement from equilibrium will leave the objective function unchanged to first order. Thus, a good measure of the loss associated with disequilibrium is given by the second order contribution to the change in the objective function. This loss may equivalently be viewed as the incentive to equilibrate. Now consider a specific path traversed by a system in its space of equilibrium states. We view such a path as a sequence of equilibrations (not necessarily discrete). The Cauchy-Schwarz inequality relates the square of the length of the path to the sum of the potential gains for equilibration along the path.

We apply this mathematical construct to the problem of measuring the welfare gains of an optimizing consumer moving along a sequence of equilibrium states. We employ the consumer surplus as our welfare measure, although Hicksian measures could also be derived. For small changes, to be sure, these various measures will agree to the order of the calculations. ${ }^{2}$ We show that the metric structure for the consumer is related to the gains in consumer surplus which accrue due to equilibration subsequent to exogenous shocks, either discrete or continuous. We explore applications of this metric structure including a measure of integrated welfare loss due to finite rate equilibrations.

\section{The metric structure defined by the utility function}

The concavity of utility as a function of the different quantities consumed guarantees that the negative of its second derivative matrix, denoted by $D^{2} U$, is positive definite. The smoothness of this utility function guarantees that $D^{2} U$ is

\footnotetext{
${ }^{2}$ In order to calculate losses in welfare using compensating or equivalent variation as measures, one would use a metric based on the second derivative of the expenditure function. Since part of our motivation is to illustrate the use of the metric structure we use the consumer's surplus to keep the arguments easy to follow. Samuelson (1983), while acknowledging the illustrative importance of the notion of consumer's surplus, prefers the Hicksian exact measures. Several authors recently argued that under certain circumstances consumer's surplus is an acceptable measure of changes in welfare. Such circumstances include the case where price changes come about through supply shocks or when the marginal utility of income is constant. Even when these conditions do not hold, consumer's surplus approximates the Hicksian measures (Dixit and Weller, 1979; Willig, 1976; Hausman, 1981; Seade, 1978; Ebert, 1984).
} 
wmmetric. If one views the equilibrium states of an economy as a manifold with cordinates given by the commodity bundle $Q=\left(q_{1}, q_{2}, \ldots, q_{n}\right)$ consumed, then $1, U$ has the properties required of a Riemannian metric on this manifold. It can then be used to define a scalar product (we omit indication of transposes)

$$
A \cdot B=A D^{2} U B
$$

.nd the length of a market velocity $\dot{Q}$

$$
\|\dot{Q}\|=\sqrt{\dot{Q} D^{2} U \dot{Q}}
$$

I sing this metric, paths $Q(t)$ on this manifold have the length $L$ given by

$$
L=\int \sqrt{\dot{Q} D^{2} U \dot{Q}} \mathrm{~d} t .
$$

Note that this integral is independent of the parameterization of the path $Q(t)$ and is a quantity dependent only on the sequence of equilibrium states. The associated notion of distance between two states is then defined to be the length of the shortest curve connecting them.

\section{A series of small discrete shocks}

We consider the case of a price-taking consumer in a market with $n$ commodities. Initial equilibrium is disturbed by a shock affecting some subset of the commodities. The source of the shock is immaterial. It could be induced by such factors as technological or institutional change, changes in market structure, or the discovery of resources. Some supply curves might be shifted down, some up. Adjustment takes time because of some friction in the system. The consumer, for instance, might be locked into a long range contract in some markets. In addition, it might take time to process information, or because of uncertainties costly information would not be acquired immediately. The process of adjustment would, however, begin immediately. Once adjustment is complete and equilibrium is reached, the sum of the areas of the consumer surplus triangles is a measure of the deadweight losses that accrue due to the fact that the adjustment was not instantaneous. We assume that the total time interval under consideration is sufficiently small that the discount rate is negligible. As a consequence we disregard the discounting of utilities over time in the summation of the welfare losses. ${ }^{3}$

\footnotetext{
${ }^{3}$ We have decided, for purposes of simplicity, to leave the discounting of welfare over time out of consideration. It would add little conceptually to the problem and would only make its solution more difficult and the results more opaque. In order to disregard it we have assumed that the duration of the process is short relative to the discount rate. To reach the geometrically significant quantities from an expression for the accrued welfare gains including the discount rate, one could apply the mean value theorem to remove the exponential term representing this discount from the integrands (summands) representing such gains. The same results follow with the inclusion of an extra term which discounts the total accrued gains to some mean time during the process.
} 
Fig. 1 depicts the consumer surplus caused by the change from equilibrium point $\left(Q_{0}, P_{0}\right)$ to $\left(Q_{\infty}, P_{\infty}\right)$. Assuming that the shock is small, the total welfare gain due to the adjustment is given by

$$
W=\int_{Q_{0}}^{Q_{\infty}}\left(P(Q)-P_{\infty}\right) \mathrm{d} Q \approx \Delta P \Delta Q / 2,
$$

where $P(Q)$ is taken along the ordinary demand curve. $Q_{\infty}$ and $P_{\infty}$ represent the equilibrium values at $t=\infty$ in the absence of new perturbations. Since $\mu P=\partial U / \partial Q$, where $\mu$ is the Lagrangian multiplier which is the marginal utility of income, and $U$ is the aggregate utility function, we have $\mu \Delta P \approx D^{2} U \Delta Q$. Thus

$$
W \approx \Delta Q D^{2} U \Delta Q / 2 \mu \text {. }
$$

The numerator may be interpreted as the square of the distance between the initial and final equilibrium states (cf. equation (3)).

The significance of the geometry emerges when we consider a series of $K$ discrete shocks and subsequent adjustments $\Delta Q_{k}, k=1, \ldots, K$. The total welfare gain is then given by one half of the sum of the squares of the steps along the path $Q(t)$ :

$$
W=\sum_{k=1}^{K} \Delta Q_{k} D^{2} U \Delta Q_{k} / 2 \mu=\sum_{k=1}^{K}\left(\Delta L_{k}\right)^{2} / 2 \mu .
$$

By the Cauchy-Schwarz inequality we get

$$
W \geq\left(\sum_{k=1}^{K} \Delta L_{k}\right)^{2} / 2 \mu K=L^{2} / 2 \mu K .
$$

Thus, the welfare gain due to the adjustment process is bounded by $1 / 2 \mu K$ times the square of the length of the path along which the small steps $\Delta Q$ are taken. Equation (7) holds with equality only for the case where all the $\Delta L$ 's are equal, i.e. when each of the shocks moves the supply curve the same distance. Note that this measures the minimum gain due to complete adjustment or, in other words, the deadweight loss which would obtain in the absence of movement toward the new equilibrium. This quantity therefore is the incentive to adjustment.

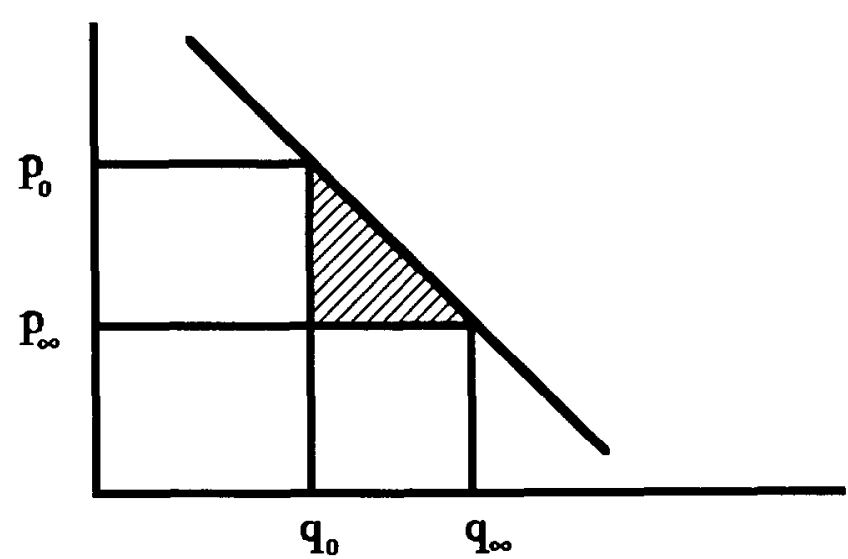

Fig. 1. The consumer surplus caused by the change from equilibrium point $\left(q_{0}, p_{0}\right)$ to $\left(q_{\infty}, p_{\infty}\right)$. 


\section{Inlegrated welfare loss}

I (I) us suppose that the market adjustment occurs over time in such a way that ifluilibrium is not reached during one period. Gains, to be sure, do accrue due to p.llial adjustment, but there exists a potential gain which is forgone on account of the slow adjustment. This forgone gain can be integrated over time until equilibrium 1. ultimately reached. We call this forgone consumer's surplus 'integrated welfare lim, $1:$

$$
I=\int_{0}^{\infty} \int_{Q(t)}^{Q_{\infty}(t)}\left[P(Q)-P_{\infty}(t)\right] \mathrm{d} Q \mathrm{~d} t .
$$

Whereas the welfare gain $W$ is the incentive to adjustment, $I$ is the total amount which could have been saved if the consumer had responded to the changes instan1.111cously. For the special case of a sequence of small discrete shocks in which each .djustment lags the shock by exactly one period, $I$ is numerically equal to $W$.

\section{Dynamics of the adjustment process}

The choice of a model for the dynamics of the adjustment to the shock becomes csisential when one considers adjustment in finite time. In his study of dynamics in cconomic equilibrium theory, Smale (1976) adopts the following form for the rate if adjustment of prices $P$ to equilibrium:

$$
D Z(P) \mathrm{d} P / \mathrm{d} t=-\lambda Z(P),
$$

where $D Z(P)$ is the matrix of first partial derivatives of the excess demand $Z$, and $\lambda$ is a constant with $\operatorname{sign} \lambda=\operatorname{sign}(\operatorname{det}[D Z])$. Equilibrium prices $P_{\infty}$ satisfy

$$
\mathrm{d} P_{\infty} / \mathrm{d} t=Z\left(P_{\infty}\right)=0 .
$$

Instead of the prices, we prefer to work with the conjugate variables $Q$. Then the excess demand is replaced by the excess prices $P-P_{\infty}$ so that the conjugate form of cquation (9) is

$$
R \mathrm{~d} Q / \mathrm{d} t=P-P_{\infty},
$$

where we have included $\lambda$ in the matrix $R$. This rate equation states that the consumption of commodities changes at a rate proportional to the potential savings, i.e. to the amounts by which prices differ from their equilibrium values. ${ }^{4}$ This can also be obtained by a linear expansion of a general dynamical law

$$
\mathrm{d} Q / \mathrm{d} t=F\left(P, P_{\infty}\right),
$$

with the property that $F(X, X)=0$ for any $X$, and where $R^{-1}$ is the Jacobian

${ }^{4}$ See, for example, Smale (1976), Gondolfo (1971), Siebrand (1979), Fisher (1983), or Beach (1957). 
matrix of $F$ with respect to $P$ evaluated at $P_{\infty}$. As before, $\mu \Delta P \approx D^{2} U \Delta Q$ so that equation (11) becomes

$$
\mathrm{d} Q / \mathrm{d} t=\mu^{-1} R^{-1} D^{2} U\left(Q-Q_{\infty}\right) .
$$

The solution to this equation is a sum of exponential decays for the components of $Q-Q_{\infty}$ along the eigendirections of $R^{-1} D^{2} U$. Although most of the derivations bclow are based on this specific form of the adjustment mechanism, the result depend only on a single parameter $\beta$ extracted from this dynamics which could formally be introduced without the dynamical law in (11). (See discussion in Section 7.) If the adjustment process can be assumed slow then, for this dynamics, $1 / \beta$ is the smallest eigenvalue of $\mu^{-1} R^{-1} D^{2} U . \beta$ is the largest lag (relaxation) time of the system.

\section{One large shock}

Let us consider $I$ for one large shock subject to the assumption of linear demand curves $P(Q)=a+b Q$. Then $D^{2} U=\mu b$ so, by equation (5),

$$
W=(1 / 2)\left(Q-Q_{\infty}\right) b\left(Q-Q_{\infty}\right) .
$$

Integration of (11) yields:

$$
Q(t)=Q_{\infty}+\left(Q_{0}-Q_{\infty}\right) \exp \left(\mu^{-1} R^{-1} D^{2} U t\right)
$$

when $R^{-1} D^{2} U$ is a constant. When substituted into (8) this yields:

$$
I=(\mu / 4)\left(Q_{0}-Q_{\infty}\right) R\left(Q_{0}-Q_{\infty}\right) .
$$

This equation states that the integrated welfare loss during the adjustment is proportional to the square of the shock times the matrix of the relaxation. Though the exact forms of (14) and (16) depend on the dynamics in (11), their general content is robust.

\section{Continuous shocks}

In an economy subject to continuous shocks $\left(P_{\infty}, Q_{\infty}\right)$, which pull the market forward, stay ahead of $(P(t), Q(t))$. This has the effect of keeping the consumer moving toward the ever changing $\left(P_{\infty}, Q_{\infty}\right)$ without ever reaching it. ${ }^{5}$ The associated welfare gain is

$$
W=\int_{Q_{0}}^{Q_{1}}\left(P-P_{\infty}\right) \mathrm{d} Q=\int_{0}^{T}(\beta / \mu)(\mathrm{d} Q / \mathrm{d} t) D^{2} U(\mathrm{~d} Q / \mathrm{d} t) \mathrm{d} t
$$

\footnotetext{
${ }^{5}$ For a previous discussion of dynamics in which the market lags equilibrium in a similar fashion see Arrow (1960).
} 
where

$$
\beta=\frac{\mu\left(P-P_{\infty}\right) \mathrm{d} Q / \mathrm{d} t}{(\mathrm{~d} Q / \mathrm{d} t) D^{2} U(\mathrm{~d} Q / \mathrm{d} t)} .
$$

Inlying the mean value theorem and the Cauchy-Schwarz inequality to the last inliplal in (17) we obtain:

$$
W=\langle\beta / \mu\rangle \int_{0}^{T}(\mathrm{~d} Q / \mathrm{d} t) D^{2} U(\mathrm{~d} Q / \mathrm{d} t) \mathrm{d} t \geq\langle\beta / \mu\rangle L^{2} / T,
$$

where $\langle\cdot\rangle$ indicates mean value, and $T$ is the total time elapsed. Note that $L$, the l.mpth of the traversed path, is an equilibrium quantity, $T$ is a constant, and the only whe of any specific dynamical model is to provide a mean value of $\beta / \mu$.

The significance of $\beta$ may be seen without recourse to dynamic models. $\beta$ is a 'lag ume' between the economy at $Q$ and its instantaneous state of equilibrium at $Q_{\infty}$. l 1 s see this note that if $P-P_{\infty}$ is small, then it can be replaced by $D^{2} U\left(Q-Q_{\infty}\right) / \mu$. 1 urthermore, if we consider the fact that at time $t_{0}$ the economy at $Q$ is heading wwward the instantaneously perceived $Q_{\infty}$, it is reasonable to assume that $Q_{\infty}\left(t_{0}\right)$ is, 11 this same approximation, the same as $Q\left(t_{0}+\gamma\right)$ for some $\gamma$. Expanding $Q$ as a Innction of $t$ linearly about $t_{0}$ we get

$$
Q_{\infty}\left(t_{0}\right)=Q\left(t_{0}+\gamma\right)=Q\left(t_{0}\right)+\gamma(\mathrm{d} Q / \mathrm{d} t)_{t_{0}} .
$$

Irom (18) we see that $\beta=\gamma$. In general, however, (20) need not be satisfied unless () ${ }_{\infty}\left(t_{0}\right)$ lies along the line through $Q\left(t_{0}\right)$ in the direction $(\mathrm{d} Q / \mathrm{d} t)_{t_{0}}$. While this will lold for sufficiently small $\gamma$, the equality of $\beta$ and $\gamma$ follows also from requiring that (20) holds with the left-hand side replaced by the perpendicular projection of () ${ }_{\infty}\left(t_{0}\right)$ onto this line, as shown in Fig. 2. 'Perpendicular' here means relative to the

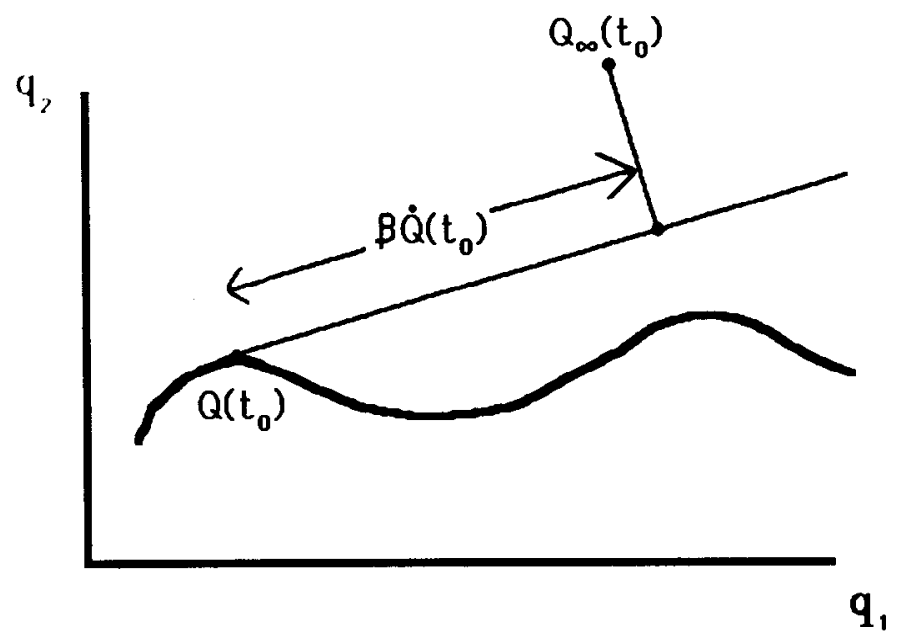

lig. 2. The perpendicular projection of the instantaneous state $Q_{\infty}\left(t_{0}\right)$ onto the tangent line to the time cvolution at $t_{0} . \beta$ can be interpreted as the lag time between the state of the economy and its instanlaneously perceived equilibrium, i.e. the projection of the equilibrium state onto the direction of movement of the economy. 
scalar product defined by the metric $D^{2} U$. This may be seen by expanding $Q_{\infty}\left(t_{0}\right)-Q\left(t_{0}\right)$ as a multiple of $(\mathrm{d} Q / \mathrm{d} t)_{t_{0}}$ and a vector perpendicular to $(\mathrm{d} Q / \mathrm{d} t)_{t_{0}}$. $\beta$ is thus generally seen to be the lag time between the state of the economy and the projection of the state with which it is trying to equilibrate onto the direction of movement of the economy. This projected state may be viewed as the state of minimum welfare loss which the consumer could reach if he were constrained to the line of his instantaneous velocity, i.e. the state of 'equilibrium' subject to the effective instantaneous constraints imposed by the direction of the tangent to his curve of evolution in the space of $Q$ 's. In this sense $\beta$ is always a lag time between the present state of the economy and the state of 'equilibrium' subject to the instantaneous effective constraints but otherwise independent of the dynamics.

We now examine $\beta$ for the dynamics described in Section 5 . Here $\beta$ is given by a Rayleigh quotient:

$$
\beta=\frac{\mu(\mathrm{d} Q / \mathrm{d} t) R(\mathrm{~d} Q / \mathrm{d} t)}{(\mathrm{d} Q / \mathrm{d} t) D^{2} U(\mathrm{~d} Q / \mathrm{d} t)}
$$

and thus $\mu / \beta$ must, at each instant, lie between the largest and smallest eigenvalues of $R^{-1} D^{2} U$ as claimed in Section 5. Furthermore, since for a slow process only the last component of $\left(Q-Q_{\infty}\right)$ is of appreciable size, $\mu / \beta$ then equals the smallest eigenvalue of $R^{-1} D^{2} U$.

Summarizing the results of this section, we showed that the welfare gains to adjustment for a continuous shock are bounded by the square of the length of the path $Q(t)$, multiplied by a mean lag time between the instantaneous states of the consumer and his instantaneous states of equilibrium, divided by the product of the total time for the process and the marginal utility of income.

\section{The geometry of the Cobb-Douglas utility function}

We consider the case of a consumer whose tastes are described by the Cobb-Douglas utility function,

$$
U(x, y, z)=x^{a} y^{b} z^{c}
$$

and discuss the geometry of the space of $Q=\{Q=(x, y, z) ; z=$ constant $\}$; for simplicity ${ }^{6}$ we will take $z=1$. The curvature associated with the Riemannian structure on this space is zero. This implies the existence of a local isometry of this manifold into the Euclidean plane. In fact, a global isometry can be constructed to

\footnotetext{
${ }^{6}$ Since the second derivative of $U$ is not strictly positive definite but possesses a direction $V$, for which $\mathrm{V} D^{2} U V=0$, we will hold one of the quantities $(z)$ constant. On this subspace $D^{2} U$ is indeed positive definite. Movement along the direction $V$ corresponds to scaling the system and has an associated length of zero.
} 
the Riemann surface of the natural logarithm of a complex variable, where each : h' 1 is given the Euclidean metric (Fig. 3). ${ }^{7}$

$A$ direct calculation shows the metric to be given by

$$
-D^{2} U[x, y]=\left[\begin{array}{ll}
a(1-a) U / x^{2} & -a b U / x y \\
-a b U / x y & b(1-b) U / y^{2}
\end{array}\right],
$$

whic we have used $[x, y]$ to indicate the coordinates in which the metric is expressal. Other coordinates may be more suited to the intrinsic geometry. Indeed, if the , inr indinates

$$
u=x^{a} y^{b}=U ; \quad v=y / x
$$

.11 used, the matrix is seen to take the diagonal form:

$$
-D^{2} U[u, v]=\left[\begin{array}{ll}
(1 / u)(1-a-b) /(a+b) & 0 \\
0 & a b /(a+b)\left(u / v^{2}\right)
\end{array}\right] .
$$

WC caution that the entries in the matrix in (25) are no longer second derivatives 1) $U$. In fact, if we temporarily denote the matrices in (23) by $M$ and in eq. (25) w $\tilde{M}$, then $\tilde{M}$ is obtained from $M$ by means of the transformation

$$
\tilde{M}=J M J,
$$

where $J$ is the Jacobian matrix $\partial(x, y) / \partial(u, v)$ and $J^{\mathrm{t}}$ is its transpose.

Equation (25) may be rewritten as an equation for the line element on the space $Q$ :

$$
\mathrm{d} s^{2}=(1-a-b) /(a+b)(1 / u) \mathrm{d} u^{2}+a b /(a+b)\left(u / v^{2}\right) \mathrm{d} v^{2}
$$

This is seen to take the form

$$
\mathrm{d} s^{2}=\mathrm{d} r^{2}+r^{2} \mathrm{~d} \theta^{2}
$$

if the usual Euclidean metric under the variable change

$$
r=2 \sqrt{(1-a-b) /(a+b) u} ; \quad \theta=1 / 2 \sqrt{a b /(1-a-b)} \ln v .
$$

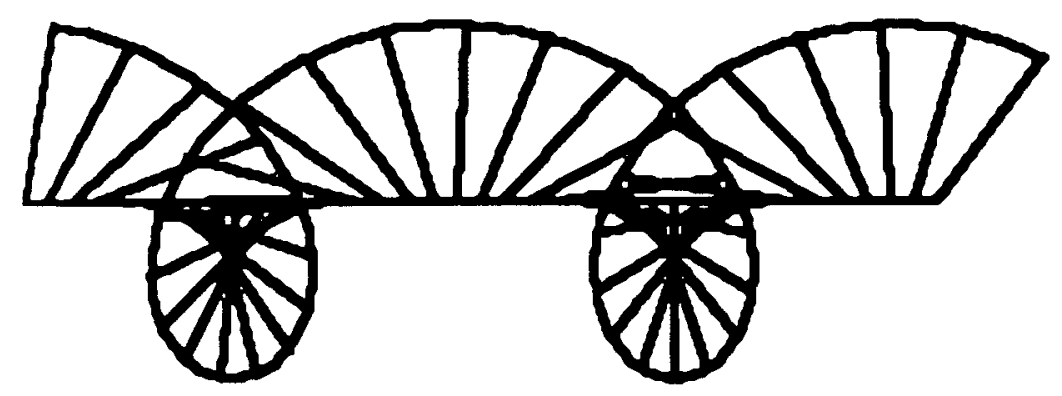

1ig. 3. An illustration of the geometry of the set of the Cobb-Douglas utility function. Locally, the surlitce is a plane although an infinite number of planes need to be spliced together for the entire surface.

\footnotetext{
${ }^{7}$ For a mathematically similar example in another discipline see the discussion of the geometry of the weal gas in Nulton and Salamon (1985).
} 
Thus, the space is locally isometric to the Euclidean plane.

Because there are in principle no boundaries on the value of $\ln (v)$, values of 1$)$ which differ by multiples of $2 \pi$ correspond to different points in the space. Fig. 3 suggests the topology of this space by showing the portion corresponding 10 $-2 \pi \leq \theta \leq 2 \pi$ and $0<r \leq 1$. Sets of states with selected values of $\theta$ are shown as rays. Because of the multisheet nature of this space the distance between two points $\left(x_{1}, y_{1}\right)$ and $\left(x_{2}, y_{2}\right)$ equals the usual expression

$$
\mathrm{d}\left[\left(x_{1}, y_{1}\right),\left(x_{2}, y_{2}\right)\right]=\sqrt{\left(x_{2}-x_{1}\right)^{2}+\left(y_{2}-y_{1}\right)^{2}}
$$

only if $\left|\theta_{1}-\theta_{2}\right| \leq \pi$. For larger angular separations the shortest curve always goes through $(0,0)$ and

$$
\mathrm{d}\left[\left(x_{1}, y_{1}\right),\left(x_{2}, y_{2}\right)\right]=\sqrt{x_{1}^{2}+y_{1}^{2}}+\sqrt{x_{2}^{2}+y_{2}^{2}} .
$$

These formulas for distance are useful particularly when the actual path $Q(t)$ traversed by the economy is not known. In this case the distances between the initial and final states $Q$ can be used in place of the length of the unknown path to give valid bounds for $W$ and $I$.

\section{Conclusions}

The purpose of this paper is to demonstrate the existence of natural metric structures on the equilibrium states of economic systems. As a first economic application, we have shown how the distances on the static demand surface can facilitatc the calculation of welfare gains which accrue to a price-taking consumer as a consequence of moving toward equilibrium. Were the consumer not a price-taker, the welfare gain due to adjustment becomes the sum of the gain due to the curvaturc of the utility function, $U$, via $D^{2} U$ and a similar term due to the curvature of the production function, $S$, via $D^{2} S$.

The non-instantaneous adjustment of the market leads to the introduction of the integrated welfare loss, $I$, which measures the total potential benefits not reaped from the evolution of the market due to finite-time response.

Finally, the geometry defined from the Cobb-Douglas utility function was shown to have zero curvature, and the natural coordinates were found in which the metric $D^{2} U$ is diagonal and the distances between states is therefore easy to calculate.

Though the results of the present paper are only preliminary, two conclusions nonetheless emerge:

(1) The inclusion of a single-parameter model of a dynamic response by a consumer to an exogenous perturbation can give useful and robust information about the response.

(2) A geometric picture of economic quantities based on the Riemannian geometry defined using the second derivative of the utility function can yield in. principle bounds on welfare gain. Such bounds can be obtained with only limited computational effort. 


\section{Acknowledgements}

We gratefully acknowledge the support of the donors of the Petroleum Research l'und administered by the American Chemical Society. J. Komlos acknowledges the financial support of an NICHD National Research Service Award 2 T32 HD07168 from the Center for Population Research. B. Andresen would like to acknowledge a grant from the Danish National Science Research Council. Finally, we would like to thank the Telluride Summer Research Center for providing us with the opportunity to work together in a stimulating environment.

\section{References}

K.J. Arrow, Price-quantity adjustments in multiple markets with rising demand, in: K.J. Arrow, S. Karlin and P. Suppes, eds., Stanford Symposium on Mathematical Methods in the Social Sciences (Stanford University Press, Stanford, 1960).

I..F. Beach, Economic Models (John Wiley and Sons, New York, 1957).

A. Deaton, The distance function in consumer behavior with applications to index numbers and optimal taxation, Rev. Econom. Stud. 46 (1979) 391-405.

A.K. Dixit and P.A. Weller, The three consumer's surpluses, Economica 46 (1979) 125-135.

U. Ebert, Exact welfare measures and economic index numbers, Zeitschrift für Nationalökonomie 44 (1984) 27-38.

F.M. Fisher, Discquilibrium Foundations of Equilibrium Economics (Cambridge University Press, Cam bridge, 1983).

I.D. Flick, P. Salamon and B. Andresen, Metric bounds on losses in adaptive coding, Information Sciences (1987) (submitted for publication).

G. Gandolfo, Mathematical Methods and Models in Equilibrium Dynamics (North-Holland, Amsterdam, 1971).

3. Gandolfo, Qualitative Analysis and Econometric Estimation of Continuous Time Dynamic Models (North-Holland, Amsterdam, 1981).

I. Hausman, Exact consumer's surplus and deadweight loss, Amer. Econom. Rev. 71 (1981) 662-676.

I.D. Nulton and P. Salamon, The geometry of the ideal gas, Phys. Rev. A31 (1985) 2520-2524.

P. Salamon and R.S. Berry, Thermodynamic length and dissipated availability, Phys. Rev. Lett. 51 (1983) 1127-1130.

P.A. Samuelson, Foundations of Economic Analysis (Harvard University Press, Cambridge, 1983), p. 197.

J. Seade, Consumer's surplus and linearity of Engel curves, The Econom. J. 88 (1978) 511-523.

I.C. Siebrand, Towards Operational Disequilibrium Macro Economics (Martinus Nijhoff, The Hague, 1979).

S. Smale, Dynamics in general equilibrium theory, Amer. Econom. Rev. 66 (1976) 288-294.

R. Willig, Consumer's surplus without apology, Amer. Econom. Rev. 66 (1976) 589-597. 